\title{
Occupational-Related Contact Dermatitis: Prevalence and Risk Factors Among Healthcare Workers in the Al'Qassim Region, Saudi Arabia During the COVID-19 Pandemic
}

Omar B. Alluhayyan ${ }^{1}$, Bashair K. Alshahri ${ }^{2}$, Abdulrahman M. Farhat ${ }^{3}$, Sulaiman Alsugair ${ }^{4}$, Jihan J. Siddiqui ${ }^{5}$, Khaled Alghabawy $^{1}$, Ghaida B. AlQefari ${ }^{1}$, Waleed O. Alolayan ${ }^{6}$, Izzat A. Abu Hashem ${ }^{7}$

1. Medicine, Qassim University, Qassim, SAU 2. Medicine, Imam Abdulrahman bin Faisal University, Khobar, SAU 3. Medicine, Sulaiman Al Rajhi University, Qassim, SAU 4. Medicine, King Saud bin Abdulaziz University for Health Sciences, Riyadh, SAU 5. Medicine, King Abdulaziz University, Jeddah, SAU 6. Nursing, Alrras General Hospital, Qassim, SAU 7. Family Medicine, Primary Health Care Center - As Sulimaniyah, Qassim, SAU

Corresponding author: Omar B. Alluhayyan, 351114266@qu.edu.sa

\section{Abstract \\ Objective}

This study aimed to estimate and investigate the prevalence and the risk factors implicated in contact dermatitis among healthcare workers in the Al'Qassim region, Saudi Arabia, during the COVID-19 pandemic.

\section{Methodology}

We conducted a cross-sectional survey among healthcare workers at hospitals in the Al'Qassim region. Data was collected using a standardized and validated Nordic Occupational Skin Questionnaire version 2002. We included 408 participants in the analysis.

\section{Results}

The majority of the respondents (66.7\%) were females. The mean age of participants was 34 (SD: \pm 9 ) years. Most of the participants who reported contact dermatitis were nurses (58.6\%). Direct patient care roles represent $78 \%$ of participants. Respondents who work $40-50$ hours per week represent $61.5 \%$ of the sample. The most commonly recorded symptoms were dryness (92.9\%), itchiness (50\%), and redness (46.4\%) of the skin. The most affected site was hand $93.5 \%$. Hand cleanser was the commonest substance implicated in the worsening of the skin changes (59.2\%). Protective glove material that worsens contact dermatitis, such as natural rubber/latex, represents $76 \%$ of responses. A significant association ( $\mathrm{p}=0.001$ )was seen in the occurrence of contact dermatitis in those with a history of allergic eye symptoms (33.3\%) and those without (58\%). Participants with a mean age of 26.47 years were more prone to develop contact dermatitis (CI: 1.197.06; $\mathrm{p}=0.067$ ). Pharmacists and interns had 3.69 and 4.90 times higher odds of having contact dermatitis (CI: 0.95-7.33; CI: $22.1 ; \mathrm{p}=0.027 ; \mathrm{p}=0.038$, respectively). Those involved in patient education and research activities at work were $6.48(\mathrm{p}=0.017)$ and $20.51(\mathrm{p}=0.024)$ times likely to develop contact dermatitis (CI:

Review began 09/20/2020 Review ended 10/09/2020 Published 10/15/2020

\section{() Copyright 2020}

Alluhayyan et al. This is an open access article distributed under the terms of the Creative Commons Attribution License CC-BY 4.0., which permits unrestricted use, distribution, and reproduction in any medium, provided the original author and source are credited.
1.38-30.31; CI: 1.49-282.15, respectively).

\section{Conclusions}

We explored the prevalence and risk factors for occupational contact dermatitis among healthcare workers in Saudi Arabia. The prevalence of reported skin changes during the pandemic was $46.4 \%$. Our study also showed that the risk factors of developing contact dermatitis include female gender, history of eye allergies, and young age group.

Categories: Dermatology, Infectious Disease

Keywords: covid-19, contact dermatitis, occupational disease, healthcare workers

\section{Introduction}

Coronavirus disease 2019 ( COVID-19) was detected in December 2019 in Wuhan, China. It is described as pneumonia of unknown cause that spreads from person to person [1]. The highly-Infectious virus spreads mainly by coughing or sneezing through droplets from the infected person, community transmission, and direct contact with contaminated objects. Appropriate use of personal protective equipment (PPE), personal hygiene, and social distancing reduce transmission rate [2]. World Health Organization (WHO) labeled COVID-19 as a global pandemic in March 2020, and all countries around the world were advised to take preventive measures to reduce the transmission of the virus [3]. In Saudi Arabia, the first case of COVID-19 was detected in a traveler on March 2nd, 2020 [4]. 
Frequent handwashing and prolonged use of PPE were observed among the healthcare workers ever since this pandemic started. Consequently, many skin diseases have emerged, such as contact dermatitis, pressure urticaria, and pressure injury. Many patients have complained about the worsening of their pre-existing skin conditions, including acne and seborrheic dermatitis [1]. Being a healthcare worker poses a high risk of developing occupational-related skin diseases [5]. Studies have shown that prolonged wearing of face mask can cause contact dermatitis, pressure urticaria, itching, acneiform eruption, and indentation. Protective caps cause scalp occlusion, which may exacerbate seborrheic dermatitis, folliculitis, and itching. According to reports, the nasal bridge is the most affected site due to the continuous, prolonged use of protective goggles. Healthcare workers frequently use latex gloves; their use contributes to blister formation, occlusion, and maceration, which may lead to contact dermatitis in certain cases. Also, excessive handwashing by detergents and disinfectants disrupts the hydrolipid barrier, which causes skin dryness and irritation. Scientists have attributed most of the skin manifestations in healthcare workers to hyperhydration, skin irritation, epidermal barrier disruption, and contact reactions that may result from prolonged use of personal protective equipment. These factors can also contribute to the worsening of preexisting skin diseases [2].

The Health and Safety Executive describes occupational skin disease as any skin disorder which is caused or worsened by work or workplace activity. Occupational skin disease is considered the second most common occupational disease. It accounts for $25 \%$ of all lost workdays [5]. Several international studies reported the prevalence of occupational skin disease in healthcare workers between 17 and 55\% [6]. Occupational contact dermatitis is responsible for 70-90\% of all occupational skin diseases and can affect the patient's quality of life. The most common symptoms include itching, swelling, blisters, cracking, or skin flaking. A crosssectional study was conducted over 12 months in Ethiopia to determine the prevalence of self-reported occupational-related contact dermatitis, indicating a prevalence of $31.5 \%$ among 422 healthcare providers [5].

Despite the problem recognition, there is insufficient data on the prevalence and risk factors for occupational-related contact dermatitis in Saudi Arabia during the COVID-19 pandemic. Therefore, we aimed to conduct a cross-sectional study to estimate the prevalence and investigate the risk factors for occupational-related contact dermatitis among the healthcare providers in the Al'Qassim region, Saudi Arabia, during the COVID-19 pandemic.

\section{Materials And Methods \\ Study design, setting, and participants}

This observational cross-sectional study was conducted from May to August 2020 in the Al'Qassim region of Saudi Arabia. The questionnaire was distributed among healthcare workers in the following four government hospitals in the Al'Qassim province: King Saud Hospital, Unaizah; Buraidah Central Hospital, Buraidah; Ar Rass General Hospital, Ar Rass; and Badai General Hospital, Badai. Also, an electronic selfadministered survey was sent specifically to healthcare workers in Al'Qassim through social media. A consecutive sampling method was considered in this study. The sample size was calculated for an assumed prevalence of $50 \%$ and an absolute precision of $5 \%$ using the statistical formula for the cross-sectional survey design. A 95\% confidence level was considered to obtain adequate power for analysis. Four hundred and eight participants were included in the study after adding a $6 \%$ non-response rate. The present study was approved by the regional bioethical committee of the Qassim region. Informed consent was gained from each patient to enroll in our study.

\section{Inclusion and exclusion criteria}

This study included healthcare workers (e.g., doctors, nurses, lab workers, other allied medical practitioners) who worked during the last four months in Al'Qassim province. All hospital administrative staff and people who had no direct contact with the patients were excluded from the study.

\section{Data collection tools and techniques}

Data was collected using a standardized Nordic Occupational Skin Questionnaire version 2002 [7]. The first part of the questionnaire was about demographic features (age, sex) and work-related questions (occupation, job hours per week, and direct or indirect contact with patients). In the second part, questions were modified to target any self-reported skin changes (i.e., dryness, redness, or itchiness) during the COVID-19 pandemic; this included affected areas of the body, number of episodes during the last two years, factors that worsen the condition (protective gloves and their type, antiseptics/disinfectants, face mask, and protective goggles), factors that improve the condition and the average time spent for hand washing and disinfecting. The last part obtained a history of any pre-existing allergic conditions and symptoms.

To ensure the quality of data obtained, we conducted a pre-test on a sample of healthcare providers before the actual data collection to test the instrument's validity and consistency, resulting in modifying some questions and misinterpretations. We also translated the questionnaire to the local language (Arabic) by experts to avoid misconceptions. 


\section{Data management and analysis}

The data were analyzed using SPSS version 25 (IBM Inc, Armonk, USA). Frequencies and percentages were calculated for categorical variables and mean \pm standard deviation (SD) for continuous variables. Association between categorical variables was analyzed using the chi-square test $(\chi 2)$. A p-value of $\leqslant 0.05$ was considered statistically significant. The co-variables were compared and contrasted across the gender of participants and the presence of self-reported occupational contact dermatitis. A univariate logistic regression analysis was performed separately for all variables significantly associated with occupational contact dermatitis to explore the associations with the dependent variable. Furthermore, a multinomial logistic regression was also performed, including all variables significantly associated with occupational contact dermatitis to identify the correlates of different variables with self-reported occupational contact dermatitis. A cut of $\leqslant 0.05 p$-value was set to evaluate the significance and odds ratios (OR) with a $95 \%$ confidence interval (CI) to establish the strength of associations. All results were summarized in tables and figures.

\section{Results}

The participants' characteristics and clinical information are presented in Table 1. More than half of the respondents were female $(66.7 \% ; \mathrm{n}=272)$. The mean age was 34 years $(\mathrm{SD} \pm 9)$. Of all the healthcare workers recruited in the study, the greatest number of respondents were the nurses $(58.6 \% ; n=239)$, doctors $(16.2 \%$; $\mathrm{n}=66)$, pharmacists $(8.3 \% ; \mathrm{n}=34)$, other occupations $(7.1 \% ; \mathrm{n}=29)$, technicians $(4.9 \% ; \mathrm{n}=20)$, and medical interns $(2.9 \% ; \mathrm{n}=12)$. Around $78 \%$ of the respondents' major activity at work involved direct patient care $(n=319)$, while the rest of the sample worked in administrative $(11 \% ; n=45)$, patient education $(5.9 \% ; n=24)$, indirect patient care $(3.7 \%$; $n=15)$ and research $(1.2 \%$; $n=5)$ roles. Out of all participates, $30 \%(n=53)$ indicated that they worked for less than 40 hours per week, 61.5\% ( $\mathrm{n}=251)$ worked $40-50$ hours per week, $18.6 \%(\mathrm{n}=76)$ worked $51-60$ hours per week, and 6.9\% worked overtime (more than 60 hours per week).

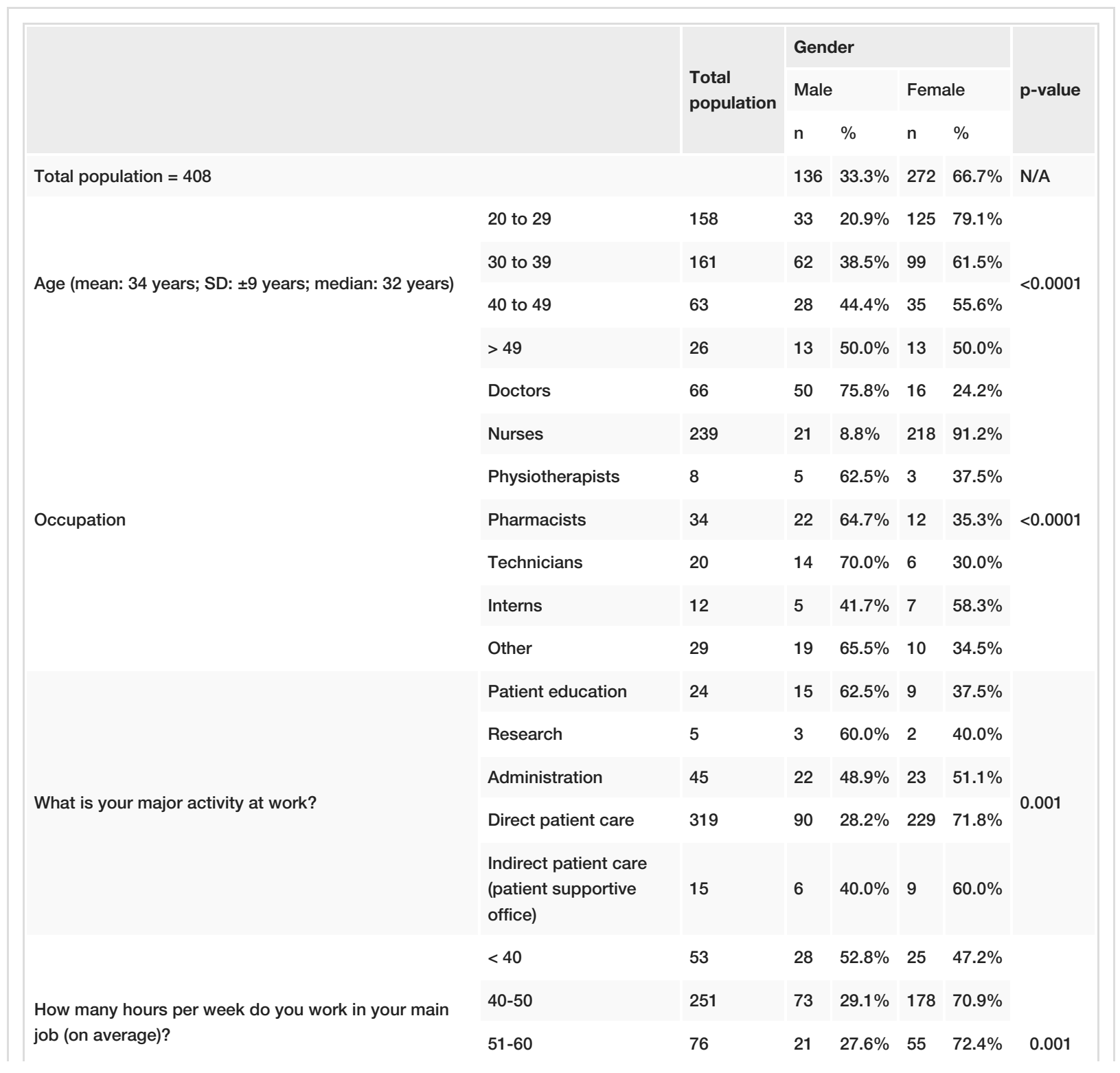




\section{Cureus}

\begin{tabular}{|llllllll} 
& $>60$ & 28 & 14 & $50.0 \%$ & 14 & $50.0 \%$ \\
$\begin{array}{l}\text { Have you ever noticed any skin changes or symptoms, } \\
\text { (i.e., dryness, redness, or itchiness) during COVID-19 }\end{array}$ & No & 219 & 87 & $39.7 \%$ & 132 & $60.3 \%$ & \\
pandemic? & Yes & 189 & 49 & $25.9 \%$ & 140 & $74.1 \%$ & 0.003 \\
& $\begin{array}{l}\text { Within the COVID 19 } \\
\text { pandemic }\end{array}$ & 134 & 36 & $26.9 \%$ & 98 & $73.1 \%$ & \\
Your skin changes initially started & $\begin{array}{l}\text { Before the COVID 19 } \\
\text { pandemic }\end{array}$ & 54 & 13 & $24.1 \%$ & 41 & $75.9 \%$ & 0.693
\end{tabular}

What do you consider as the most important things in the workplace that worsen your skin changes?

\begin{tabular}{|c|c|c|c|c|c|c|c|}
\hline \multirow{2}{*}{ Protective gloves } & No & 109 & 29 & $26.6 \%$ & 80 & $73.4 \%$ & \multirow{2}{*}{0.847} \\
\hline & Yes & 75 & 19 & $25.3 \%$ & 56 & $74.7 \%$ & \\
\hline \multirow{2}{*}{ Hand cleanser, soap } & No & 75 & 29 & $38.7 \%$ & 46 & $61.3 \%$ & \multirow{2}{*}{0.001} \\
\hline & Yes & 109 & 19 & $17.4 \%$ & 90 & $82.6 \%$ & \\
\hline \multirow{2}{*}{ Antiseptics/disinfectants } & No & 96 & 27 & $28.1 \%$ & 69 & $71.9 \%$ & \multirow{2}{*}{0.511} \\
\hline & Yes & 88 & 21 & $23.9 \%$ & 67 & $76.1 \%$ & \\
\hline \multirow{2}{*}{ Face mask } & No & 126 & 36 & $28.6 \%$ & 90 & $71.4 \%$ & \multirow{2}{*}{0.258} \\
\hline & Yes & 58 & 12 & $20.7 \%$ & 46 & $79.3 \%$ & \\
\hline \multirow{2}{*}{ Protective goggles } & No & 180 & 47 & $26.1 \%$ & 133 & $73.9 \%$ & \multirow{2}{*}{0.960} \\
\hline & Yes & 4 & 1 & $25.0 \%$ & 3 & $75.0 \%$ & \\
\hline \multirow{2}{*}{ None of the above } & No & 178 & 47 & $26.4 \%$ & 131 & $73.6 \%$ & \multirow{2}{*}{0.593} \\
\hline & Yes & 6 & 1 & $16.7 \%$ & 5 & $83.3 \%$ & \\
\hline
\end{tabular}

What do you consider as the most important things outside the workplace that worsen your skin changes?

Frequent hand washing

Protective gloves

None of the above

Questions

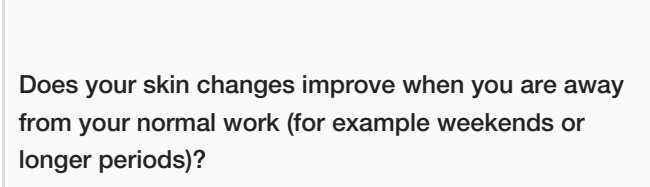

How many times do you wash your hands during a usual working day before the COVID 19 pandemic?

How many times do you wash your hands during a usual working day during the COVID 19 pandemic?

\begin{tabular}{llllllll} 
No & 90 & 32 & $35.6 \%$ & 58 & $64.4 \%$ & 0.001 \\
Yes & 89 & 12 & $13.5 \%$ & 77 & $86.5 \%$ & \\
No & 138 & 33 & $23.9 \%$ & 105 & $76.1 \%$ & \\
Yes & 41 & 11 & $26.8 \%$ & 30 & $73.2 \%$ & 0.703 \\
No & 168 & 40 & $23.8 \%$ & 128 & $76.2 \%$ & 0.349 \\
Yes & 11 & 4 & $36.4 \%$ & 7 & $63.6 \%$ & \\
& & & & & & \\
No & 11 & 4 & $36.4 \%$ & 7 & $63.6 \%$ & \\
Yes, sometimes & 82 & 17 & $20.7 \%$ & 65 & $79.3 \%$ & \\
Yes, usually & 78 & 20 & $25.6 \%$ & 58 & $74.4 \%$ & 0.523 \\
Don't know & 8 & 3 & $37.5 \%$ & 5 & $62.5 \%$ & \\
0-5 times per day & 105 & 51 & $48.6 \%$ & 54 & $51.4 \%$ & \\
6-10 times per day & 141 & 59 & $41.8 \%$ & 82 & $58.2 \%$ & \\
11-20 times per day & 89 & 13 & $14.6 \%$ & 76 & $85.4 \%$ & $<0.0001$ \\
more than 20 times per & 73 & 13 & $17.8 \%$ & 60 & $82.2 \%$ & \\
day & & 13 & $52.0 \%$ & 12 & $48.0 \%$ & \\
0-5 times per day & 25 & 50 & $50.5 \%$ & 49 & $49.5 \%$ & \\
6-10 times per day & 99 & 34 & $30.9 \%$ & 76 & $69.1 \%$ & $<0.0001$ \\
\hline 11-20 times per day & 110 & & & & & & \\
\hline
\end{tabular}




\section{Cureus}

\begin{tabular}{|c|c|c|c|c|c|c|c|}
\hline & $\begin{array}{l}\text { more than } 20 \text { times per } \\
\text { day }\end{array}$ & 174 & 39 & $22.4 \%$ & 135 & $77.6 \%$ & \\
\hline \multirow{3}{*}{$\begin{array}{l}\text { How much time do you spend on each hand washing } \\
\text { before the COVID } 19 \text { pandemic? }\end{array}$} & Less than 40 seconds & 180 & 76 & $42.2 \%$ & 104 & $57.8 \%$ & \multirow{3}{*}{0.001} \\
\hline & $40-50$ seconds & 175 & 51 & $29.1 \%$ & 124 & $70.9 \%$ & \\
\hline & More than 50 seconds & 53 & 9 & $17.0 \%$ & 44 & $83.0 \%$ & \\
\hline \multirow{3}{*}{$\begin{array}{l}\text { How much time do you spend on each hand washing } \\
\text { during the COVID } 19 \text { pandemic? }\end{array}$} & Less than 40 seconds & 62 & 30 & $48.4 \%$ & 32 & $51.6 \%$ & \multirow{3}{*}{0.006} \\
\hline & $40-50$ seconds & 226 & 76 & $33.6 \%$ & 150 & $66.4 \%$ & \\
\hline & More than 50 seconds & 120 & 30 & $25.0 \%$ & 90 & $75.0 \%$ & \\
\hline \multirow{4}{*}{$\begin{array}{l}\text { How many times do you use disinfecting alcohol for } \\
\text { hands in a usual working day before the COVID } 19 \\
\text { pandemic? }\end{array}$} & $0-5$ times per day & 145 & 74 & $51.0 \%$ & 71 & $49.0 \%$ & \multirow{4}{*}{$<0.0001$} \\
\hline & 6-10 times per day & 118 & 40 & $33.9 \%$ & 78 & $66.1 \%$ & \\
\hline & $11-20$ times per day & 90 & 14 & $15.6 \%$ & 76 & $84.4 \%$ & \\
\hline & $\begin{array}{l}\text { more than } 20 \text { times per } \\
\text { day }\end{array}$ & 55 & 8 & $14.5 \%$ & 47 & $85.5 \%$ & \\
\hline \multirow{4}{*}{$\begin{array}{l}\text { How many times do you use disinfecting alcohol for } \\
\text { hands in a usual working day during the COVID } 19 \\
\text { pandemic? }\end{array}$} & $0-5$ times per day & 25 & 17 & $68.0 \%$ & 8 & $32.0 \%$ & \multirow{4}{*}{$<0.0001$} \\
\hline & 6-10 times per day & 107 & 50 & $46.7 \%$ & 57 & $53.3 \%$ & \\
\hline & 11-20 times per day & 121 & 33 & $27.3 \%$ & 88 & $72.7 \%$ & \\
\hline & $\begin{array}{l}\text { more than } 20 \text { times per } \\
\text { day }\end{array}$ & 155 & 36 & $23.2 \%$ & 119 & $76.8 \%$ & \\
\hline
\end{tabular}

\section{TABLE 1: Comparison of participants characteristics by gender in a sample of healthcare providers}

Table 2 shows that the overall prevalence of self-reported skin changes (i.e., dryness, redness, or itchiness) during the COVID-19 pandemic was $46.3 \%(\mathrm{n}=189)$. The most commonly reported types of skin changes were dryness (92.9\%; $n=78)$, itchiness (50\%; $n=42)$, and redness $(46.4 \%$; $n=39$ ), as indicated in Figure 1 . Hands, nasal bridge, and wrists or forearms (excluding fronts of elbows) ranked as the three most commonly affected areas, as reported by $93.5 \%(\mathrm{n}=172), 21.7 \%(\mathrm{n}=40)$, and $15.2 \%(\mathrm{n}=28)$ respondents respectively (Figure 2). The three most commonly reported factors in the workplace that worsen the participants' skin changes were hand cleanser/soap (59.2\%; $\mathrm{n}=109)$, antiseptic/disinfectants $47.8 \% ; \mathrm{n}=88)$, and protective gloves $(2.2 \% ; \mathrm{n}=75)$. Respondents reported that $76 \%(\mathrm{n}=57)$ wear natural rubber/latex gloves, $16 \%(\mathrm{n}=12)$ wear synthetic rubber gloves, and $12 \%(\mathrm{n}=9)$ wear plastic gloves. A significant association $(\mathrm{p}=0.001)$ was seen in the occurrence of contact dermatitis between those who had a personal history suggestive of allergic eye symptoms (itching, watery, red, or swollen eyes, e.g., from pollen or animals) reported by $33.3 \%(n=23)$, and those who had not $(58 \%$; $n=188)$.

\begin{tabular}{|c|c|c|c|c|c|c|}
\hline & & \multicolumn{4}{|c|}{$\begin{array}{l}\text { Have you ever noticed any skin changes or } \\
\text { symptoms, (i.e. dryness, redness, or itchiness) } \\
\text { during COVID-19 pandemic? }\end{array}$} & \multirow{3}{*}{$\begin{array}{l}p- \\
\text { value }\end{array}$} \\
\hline & & \multicolumn{2}{|l|}{ No } & \multicolumn{2}{|l|}{ Yes } & \\
\hline & & $\mathrm{n}$ & $\%$ & $\mathrm{n}$ & $\%$ & \\
\hline \multirow{2}{*}{ Gender } & Male & 87 & $64.0 \%$ & 49 & $36.0 \%$ & \multirow{2}{*}{0.003} \\
\hline & Female & 132 & $48.5 \%$ & 140 & $51.5 \%$ & \\
\hline \multirow{5}{*}{ Age } & 20 to 29 & 69 & $43.7 \%$ & 89 & $56.3 \%$ & \multirow{4}{*}{0.000} \\
\hline & 30 to 39 & 86 & $53.4 \%$ & 75 & $46.6 \%$ & \\
\hline & 40 to 49 & 46 & $73.0 \%$ & 17 & $27.0 \%$ & \\
\hline & $>49$ & 18 & $69.2 \%$ & 8 & $30.8 \%$ & \\
\hline & Doctors & 36 & $54.5 \%$ & 30 & $45.5 \%$ & \\
\hline
\end{tabular}




\begin{tabular}{|c|c|c|c|c|c|c|}
\hline \multirow{6}{*}{ Occupation } & Nurses & 128 & $53.6 \%$ & 111 & $46.4 \%$ & \multirow{6}{*}{0.040} \\
\hline & Physiotherapists & 6 & $75.0 \%$ & 2 & $25.0 \%$ & \\
\hline & Pharmacists & 13 & $38.2 \%$ & 21 & $61.8 \%$ & \\
\hline & Technicians & 15 & $75.0 \%$ & 5 & $25.0 \%$ & \\
\hline & Interns & 3 & $25.0 \%$ & 9 & $75.0 \%$ & \\
\hline & Other & 18 & $62.1 \%$ & 11 & $37.9 \%$ & \\
\hline \multirow{5}{*}{ What is your major activity at work? } & Patient education & 9 & $37.5 \%$ & 15 & $62.5 \%$ & \multirow{5}{*}{0.021} \\
\hline & Research & 1 & $20.0 \%$ & 4 & $80.0 \%$ & \\
\hline & Administration & 32 & $71.1 \%$ & 13 & $28.9 \%$ & \\
\hline & Direct patient care & 167 & $52.4 \%$ & 152 & $47.6 \%$ & \\
\hline & $\begin{array}{l}\text { Indirect patient } \\
\text { care (patient } \\
\text { supportive office) }\end{array}$ & 10 & $66.7 \%$ & 5 & $33.3 \%$ & \\
\hline \multirow{4}{*}{$\begin{array}{l}\text { How many hours per week do you work in your } \\
\text { main job (on average)? }\end{array}$} & $<40$ & 25 & $47.2 \%$ & 28 & $52.8 \%$ & \multirow{4}{*}{0.197} \\
\hline & $40-50$ & 145 & $57.8 \%$ & 106 & $42.2 \%$ & \\
\hline & $51-60$ & 37 & $48.7 \%$ & 39 & $51.3 \%$ & \\
\hline & $>60$ & 12 & $42.9 \%$ & 16 & $57.1 \%$ & \\
\hline \multirow{4}{*}{$\begin{array}{l}\text { How many times do you wash your hands } \\
\text { during a usual working day before the COVID } \\
19 \text { pandemic? }\end{array}$} & $0-5$ times per day & 56 & $53.3 \%$ & 49 & $46.7 \%$ & \multirow{4}{*}{0.237} \\
\hline & 6-10 times per day & 67 & $47.5 \%$ & 74 & $52.5 \%$ & \\
\hline & $\begin{array}{l}\text { 11-20 times per } \\
\text { day }\end{array}$ & 52 & $58.4 \%$ & 37 & $41.6 \%$ & \\
\hline & $\begin{array}{l}\text { more than } 20 \text { times } \\
\text { per day }\end{array}$ & 44 & $60.3 \%$ & 29 & $39.7 \%$ & \\
\hline \multirow{4}{*}{$\begin{array}{l}\text { How many times do you wash your hands } \\
\text { during a usual working day during the COVID } \\
19 \text { pandemic? }\end{array}$} & $0-5$ times per day & 14 & $56.0 \%$ & 11 & $44.0 \%$ & \multirow{4}{*}{0.738} \\
\hline & 6-10 times per day & 57 & $57.6 \%$ & 42 & $42.4 \%$ & \\
\hline & $\begin{array}{l}\text { 11-20 times per } \\
\text { day }\end{array}$ & 55 & $50.0 \%$ & 55 & $50.0 \%$ & \\
\hline & $\begin{array}{l}\text { more than } 20 \text { times } \\
\text { per day }\end{array}$ & 93 & $53.4 \%$ & 81 & $46.6 \%$ & \\
\hline \multirow{3}{*}{$\begin{array}{l}\text { How much time do you spend on each hand } \\
\text { washing before the COVID } 19 \text { pandemic? }\end{array}$} & $\begin{array}{l}\text { Less than } 40 \\
\text { seconds }\end{array}$ & 87 & $48.3 \%$ & 93 & $51.7 \%$ & \multirow{3}{*}{0.110} \\
\hline & $40-50$ seconds & 104 & $59.4 \%$ & 71 & $40.6 \%$ & \\
\hline & $\begin{array}{l}\text { More than } 50 \\
\text { seconds }\end{array}$ & 28 & $52.8 \%$ & 25 & $47.2 \%$ & \\
\hline \multirow{3}{*}{$\begin{array}{l}\text { How much time do you spend on each hand } \\
\text { washing during the COVID } 19 \text { pandemic? }\end{array}$} & $\begin{array}{l}\text { Less than } 40 \\
\text { seconds }\end{array}$ & 35 & $56.5 \%$ & 27 & $43.5 \%$ & \multirow{3}{*}{0.790} \\
\hline & $40-50$ seconds & 118 & $52.2 \%$ & 108 & $47.8 \%$ & \\
\hline & $\begin{array}{l}\text { More than } 50 \\
\text { seconds }\end{array}$ & 66 & $55.0 \%$ & 54 & $45.0 \%$ & \\
\hline \multirow{4}{*}{$\begin{array}{l}\text { How many times do you use disinfecting } \\
\text { alcohol for hands in a usual working day before } \\
\text { the COVID } 19 \text { pandemic? }\end{array}$} & $0-5$ times per day & 72 & $49.7 \%$ & 73 & $50.3 \%$ & \multirow{4}{*}{0.287} \\
\hline & 6-10 times per day & 60 & $50.8 \%$ & 58 & $49.2 \%$ & \\
\hline & $\begin{array}{l}\text { 11-20 times per } \\
\text { day }\end{array}$ & 53 & $58.9 \%$ & 37 & $41.1 \%$ & \\
\hline & $\operatorname{lan} 20$ & 34 & $61.8 \%$ & 21 & $38.2 \%$ & \\
\hline
\end{tabular}




\section{Cureus}

How many times do you use disinfecting

alcohol for hands in a usual working day during

the COVID 19 pandemic?

\begin{tabular}{|c|c|c|c|c|}
\hline $0-5$ times per day & 16 & $64.0 \%$ & 9 & $36.0 \%$ \\
\hline 6-10 times per day & 58 & $54.2 \%$ & 49 & $45.8 \%$ \\
\hline $\begin{array}{l}\text { 11-20 times per } \\
\text { day }\end{array}$ & 61 & $50.4 \%$ & 60 & $49.6 \%$ \\
\hline $\begin{array}{l}\text { more than } 20 \text { times } \\
\text { per day }\end{array}$ & 84 & $54.2 \%$ & 71 & $45.8 \%$ \\
\hline
\end{tabular}

TABLE 2: Prevalence of skin changes by multiple factors among a sample of healthcare providers

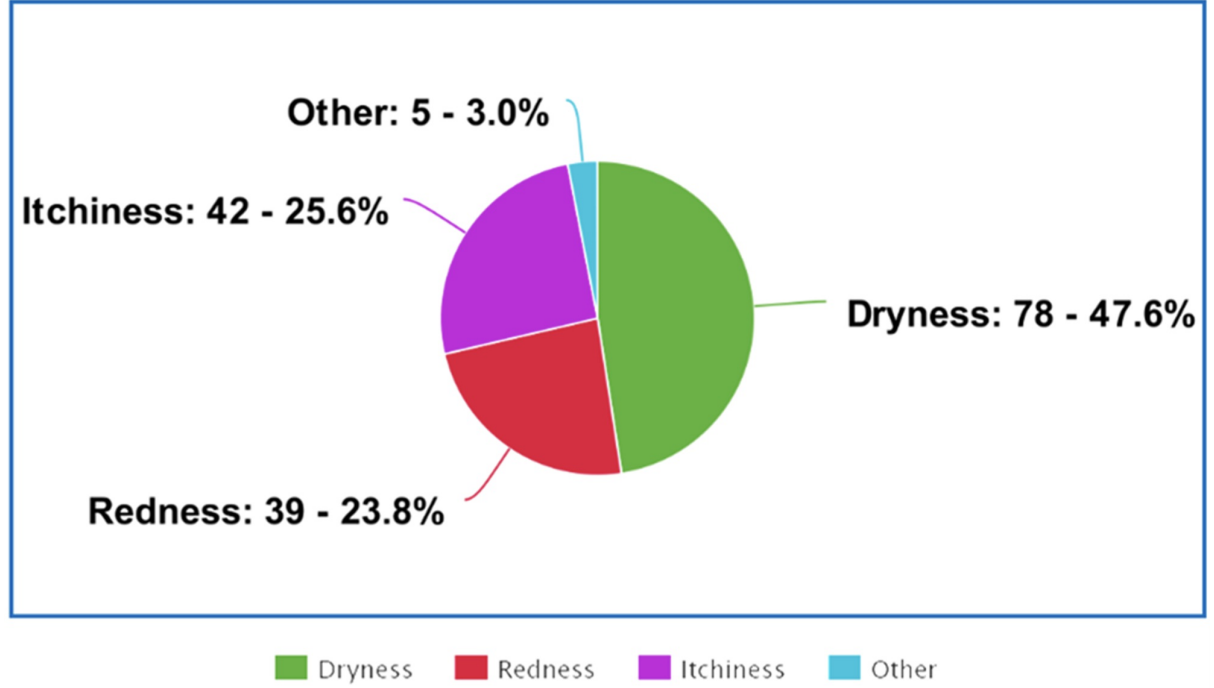

FIGURE 1: Distribution of the reported skin changes

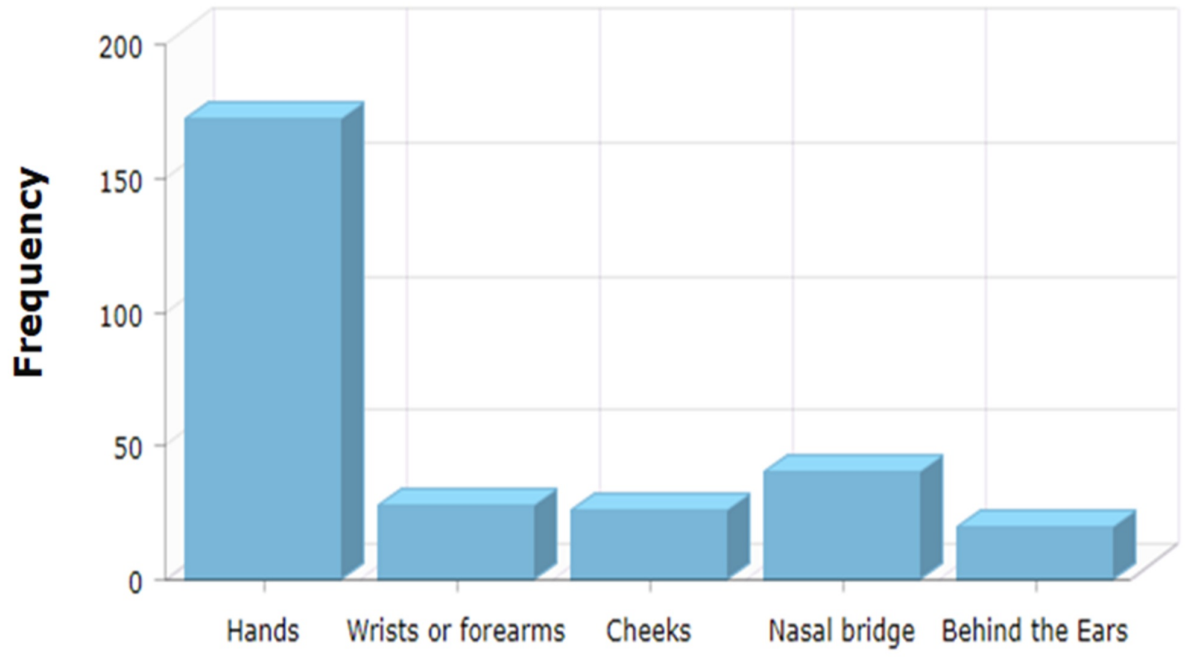

Affected areas

FIGURE 2: Frequency of the affected areas

\section{Risk factors for contact dermatitis among healthcare workers}

Univariate logistic regression showed that contact dermatitis was significantly associated with several demographic characteristics and work conditions (Table 3). Female sex was associated with a higher 


\section{Cureus}

prevalence $(\mathrm{p}=0.003)$. Participant age was also an important factor $(\mathrm{p}=0.019)$. Among the diverse occupational roles, medical interns were the most frequently affected group by contact dermatitis $(\mathrm{p}=0.038)$. A multivariate logistic regression analysis (Table 3), showed that female healthcare workers had 2.36 times the odds of reporting contact dermatitis than their male counterparts (95\% CI: 1.23-1.23; $\mathrm{p}=0.008$ ). Participants in the age group of $20-29$ years with a mean of 26.47 years were more likely to develop contact dermatitis, compared to the other age groups (95\% CI: 1.19-7.06; p=0.067). Pharmacists and interns had 3.69 and 4.90 times higher odds to have contact dermatitis than other occupations (95\% CI: 0.95-7.33; 95\% CI: 22.1; p=0.027, p=0.038, respectively). Those respondents who were involved in patient education and research activity at work were $6.48(\mathrm{p}=0.017)$ and $20.51(\mathrm{p}=0.024)$ times likely to develop contact dermatitis, compared to respondents with other roles (95\% CI: 1.38-30.31; 95\% CI: 1.49-282.15, respectively).

\begin{tabular}{|c|c|c|c|c|c|}
\hline \multicolumn{2}{|l|}{ Variables $(n=408)$} & $\begin{array}{l}\text { Model } 1^{\text {a }} \\
\text { OR }(95 \% \\
\text { Cl) }\end{array}$ & $\begin{array}{l}\text { p- } \\
\text { value }\end{array}$ & $\begin{array}{l}\text { Model } 2^{b} \\
\text { OR }(95 \% \\
\text { Cl) }\end{array}$ & $\begin{array}{l}\text { p- } \\
\text { value }\end{array}$ \\
\hline \multirow[t]{2}{*}{ Gender } & Female & $\begin{array}{l}1.88(1.23- \\
1.23)\end{array}$ & $0.003^{*}$ & $\begin{array}{l}2.36(1.25 \\
-4.45)\end{array}$ & $0.008^{\star}$ \\
\hline & Male & Ref & Ref & Ref & Ref \\
\hline \multirow{4}{*}{ Age } & 20 to 29 & $\begin{array}{l}2.90(1.19- \\
7.06)\end{array}$ & $0.019^{*}$ & $\begin{array}{l}2.49(0.93 \\
-6.63)\end{array}$ & 0.067 \\
\hline & 30 to 39 & $\begin{array}{l}1.96(0.80- \\
4.77)\end{array}$ & 0.131 & $\begin{array}{l}2.14(0.81 \\
-5.63)\end{array}$ & 0.120 \\
\hline & 40 to 49 & $\begin{array}{l}0.83(0.30- \\
2.26)\end{array}$ & 0.718 & $\begin{array}{l}1.00(0.34 \\
-2.92)\end{array}$ & 0.988 \\
\hline & $>49$ & Ref & Ref & Ref & Ref \\
\hline \multirow{7}{*}{ Occupation } & Doctors & $\begin{array}{l}1.36(0.55- \\
3.33)\end{array}$ & 0.496 & $\begin{array}{l}1.93(0.67 \\
-5.56)\end{array}$ & 0.219 \\
\hline & Nurses & $\begin{array}{l}1.41(0.64- \\
3.13)\end{array}$ & 0.386 & $\begin{array}{l}1.20(0.45 \\
-3.22\end{array}$ & 0.704 \\
\hline & Physiotherapists & $\begin{array}{l}0.54(0.09- \\
3.19)\end{array}$ & 0.501 & $\begin{array}{l}0.71(0.10 \\
-4.81)\end{array}$ & 0.732 \\
\hline & Pharmacists & $\begin{array}{l}2.64(0.95- \\
7.33)\end{array}$ & 0.062 & $\begin{array}{l}3.69(1.15 \\
-11.81)\end{array}$ & $0.027^{*}$ \\
\hline & Technicians & $\begin{array}{l}0.54(0.15- \\
1.92)\end{array}$ & 0.346 & $\begin{array}{l}0.57(0.13 \\
-2.34)\end{array}$ & 0.436 \\
\hline & Interns & $\begin{array}{l}4.90(1.08- \\
22.1)\end{array}$ & $0.038^{\star}$ & $\begin{array}{l}4.32(0.79 \\
-23.46)\end{array}$ & 0.089 \\
\hline & Other & Ref & Ref & Ref & Ref \\
\hline \multirow{6}{*}{ What is your major activity at work? } & Patient education & $\begin{array}{l}3.33(0.86- \\
12.9)\end{array}$ & 0.082 & $\begin{array}{l}6.48(1.38 \\
-30.31)\end{array}$ & $0.017^{*}$ \\
\hline & Research & $\begin{array}{l}8.00(0.69- \\
91.7)\end{array}$ & 0.095 & $\begin{array}{l}20.51 \\
(1.49- \\
282.15)\end{array}$ & $0.024^{\star}$ \\
\hline & Administration & $\begin{array}{l}0.81(0.23- \\
2.84)\end{array}$ & 0.745 & $\begin{array}{l}2.29(0.54 \\
-9.75)\end{array}$ & 0.260 \\
\hline & Direct patient care & $\begin{array}{l}1.82(0.60- \\
5.44)\end{array}$ & 0.28 & $\begin{array}{l}3.04(0.84 \\
-10.97)\end{array}$ & 0.089 \\
\hline & $\begin{array}{l}\text { Indirect patient care } \\
\text { (patient supportive } \\
\text { office) }\end{array}$ & Ref & Ref & Ref & Ref \\
\hline & No & $\begin{array}{l}0.82(0.29- \\
2.33)\end{array}$ & 0.119 & $\begin{array}{l}0.93(0.31 \\
-2.84)\end{array}$ & 0.911 \\
\hline
\end{tabular}




\section{Cureus}

\begin{tabular}{|c|c|c|c|c|c|}
\hline \multirow[t]{2}{*}{$\begin{array}{l}\text { Have your eyes ever shown allergic symptoms Itching, } \\
\text { Watery, red or swollen eyes, e.g., from pollens or animals? }\end{array}$} & Yes & $\begin{array}{l}2.28(0.73- \\
7.08)\end{array}$ & 0.152 & $\begin{array}{l}3.03(0.89 \\
-10.27)\end{array}$ & 0.074 \\
\hline & Don't know & Ref & Ref & Ref & Ref \\
\hline${ }^{*}$ Not the reference category & & & & & \\
\hline a: unadjusted (crude) OR & & & & & \\
\hline b: complete model: multinomial multivariate logistic regression & & & & & \\
\hline
\end{tabular}

\section{Discussion}

Our primary goal of this study was to estimate the prevalence and investigate the risk factors for occupational-related contact dermatitis among healthcare workers in Al'Qassim Region, Saudi Arabia, during the Covid-19 pandemic.

Our study shows that the commonly reported symptom is skin dryness (92.9\%), whereas an Ethiopian study was done in 2018 showed the majority of the participants reported skin redness (28.6\%) [5]. However, the hands are the most affected area in both studies. Moreover, both studies show that nurses are more likely to suffer from skin changes than any other healthcare provider. It can be due to the fact that the majority of the participants were nurses, and nurses have more direct contact with patients. A study published in 2020 stated that an increase in hand hygiene could lead to skin changes involving skin dryness [3]. The pandemic undoubtedly has increased the necessity of frequent hand hygiene, thus increasing the unwanted skin changes.

Our study shows that the majority (76\%) of healthcare workers wear latex gloves. Surprisingly, another study done during the pandemic in Hubei Province's hospitals found the adverse effects of latex gloves on the skin [8]. Our results show that the most commonly reported symptom is skin dryness (92.9\%). Similarly, the Hubei study results reported the most common symptom is skin dryness (55.8\%). Moreover, both of these studies show the second most commonly reported symptom is skin itchiness - $50 \%$ and $31.2 \%$, respectively. Skin itchiness could be an undiagnosed latex allergy since it is one of the symptoms of the disease. A study done in 2014 claims that healthcare providers are at high risk of developing latex allergy due to the increase in using latex gloves, the duration of wearing latex gloves, and exposure to the high concentration of latex antigens [9]. Our study shows that plastic gloves can cause less skin irritation than any other type of gloves (12\%), whereas the Hubei study shows that none had reported any skin irritation when wearing plastic gloves [8].

Interestingly, our results show that females are more prone to develop contact dermatitis. Nevertheless, a study done in Singapore during severe acute respiratory syndrome (SARS) showed no gender predisposition, but another study published in 2016 claims that females are at a higher risk of developing facial and nonfacial contact dermatitis $[10,11]$. Moreover, our study shows that pharmacists and medical interns have a higher chance of developing contact dermatitis. On the other hand, the Singaporean study shows no occupation would be at a higher risk. We included in our study $58.6 \%$ of nurses, $16.2 \%$ of doctors, $8.3 \%$ of pharmacists, $4.9 \%$ of technicians, $2.9 \%$ of medical interns, $5.9 \%$ of patient education employees, and $1.2 \%$ of researchers, but they only included $14.3 \%$ of doctors, $73 \%$ of nurses, and $12.7 \%$ of clinic assistants and counter clerks. It is hard to conclude which occupation is at higher risk of developing skin changes during the pandemic due to the limitation of having equal representative numbers from each occupation. Our results and their results show that the young age group is more susceptible to developing contact dermatitis with a mean age of 26.4 years and 28.7 years, respectively. A study done in Riyadh states that elderly workers are less likely to have allergic contact dermatitis due to the age-related decrease in their inflammatory response [12].

Our study has some limitations. It does not cover all the hospitals, including private hospitals, in the Al'Qassim region. Also, not all the participants completely filled the questionnaire.

\section{Conclusions}

Personal protective equipment has been markedly used during the COVID-19 pandemic, which led to skin manifestations among healthcare workers. In this study, we explored the prevalence and risk factors for occupational contact dermatitis among healthcare workers in Saudi Arabia. Our study showed risk factors for developing contact dermatitis among health care workers, which include a personal history of eye allergies, female gender, and young age. Also, skin symptoms have increased during the COVID-19 pandemic. The most commonly reported skin irritant was hand cleanser, followed by disinfectants and protective gloves. Among the healthcare workers, skin dryness was the highest prevalence, with $92.9 \%$. 


\section{Additional Information}

Disclosures

Human subjects: Consent was obtained by all participants in this study. Animal subjects: All authors have confirmed that this study did not involve animal subjects or tissue. Conflicts of interest: In compliance with the ICMJE uniform disclosure form, all authors declare the following: Payment/services info: All authors have declared that no financial support was received from any organization for the submitted work. Financial relationships: All authors have declared that they have no financial relationships at present or within the previous three years with any organizations that might have an interest in the submitted work. Other relationships: All authors have declared that there are no other relationships or activities that could appear to have influenced the submitted work.

\section{References}

1. Darlenski R, Tsankov N: COVID-19 pandemic and the skin: what should dermatologists know? (IN PRESS). Clin Dermatol. 2020, 10.1016/j.clindermatol.2020.03.012

2. Masood S, Tabassum S, Naveed S, Jalil P: COVID-19 Pandemic \& skin care guidelines for health care professionals. Pak J Med Sci. 2020, 36: 10.12669/pjms.36.COVID19-S4.2748

3. Beiu C, Mihai M, Popa L, Cima L, Popescu M: Frequent hand washing for COVID-19 prevention can cause hand dermatitis: management tips. Cureus. 2020, 12:e7506. 10.7759/cureus.7506

4. Algaissi A, Alharbi N, Hassanain M, Hashem A: Preparedness and response to COVID-19 in Saudi Arabia: building on MERS experience. J Infect Public Health. 2020, 13:834-838. 10.1016/j.jiph.2020.04.016

5. Mekonnen T, Yenealem D, Tolosa B: Self-report occupational-related contact dermatitis: prevalence and risk factors among healthcare workers in Gondar town, Northwest Ethiopia, 2018 - a cross-sectional study. Environ Health Prev Med. 2019, 24:10.1186/s12199-019-0765-0

6. Campion K: A survey of occupational skin disease in UK health care workers . Occup Med. 2015, 65:29-31. 10.1093/occmed/kqu170

7. Susitaival P, Flyvholm MA, Meding B, et al.: Nordic Occupational Skin Questionnaire (NOSQ- 2002): A new tool for surveying occupational skin diseases and exposure. Contact Derm. 2003, 49:70-76. 10.1111/j.01051873.2003.00159.x

8. Hu K, Fan J, Li X, Gou X, Li X, Zhou X: The adverse skin reactions of health care workers using personal protective equipment for COVID-19. Medicine. 2020 , 12:e20603. 10.1097/MD.0000000000020603

9. Zak HN, Kaste LM, Schwarzenberger K, Barry MJ, Galbraith GM: Health-care workers and latex allergy. Arch Environ Health: Int J. 2000, 1:336-46. 10.1080/00039890009604026

10. Foo CC, Goon AT, Leow YH, Goh CL: Adverse skin reactions to personal protective equipment against severe acute respiratory syndrome-a descriptive study in Singapore. Contact Derm. 2006, 55:291-294. 10.1111/j.1600-0536.2006.00953.x

11. Kasemsarn $\mathrm{P}$, Iamphonrat $\mathrm{T}$, Boonchai $\mathrm{W}$ : Risk factors and common contact allergens in facial allergic contact dermatitis patients. Int J Dermatol. 2016, 55:417-424. 10.1111/ijd.12880

12. Al-Sheikh OA, El-Rab MO: Allergic contact dermatitis: clinical features and profile of sensitizing allergens . Int J Dermatol. 1996, 35:493-497. 10.1111/j.1365-4362.1996.tb01664.x 